Hiroki Chikumi • Toshiyuki Yamamoto $\cdot$ Yasutoshi Ohta

Eiji Nanba $\cdot$ Keiko Nagata $\cdot$ Haruaki Ninomiya

Kohshi Narasaki • Tatsuo Katoh • Ichiro Hisatome

Kimiyo Ono $\cdot$ Noriyuki Tanaka $\cdot$ Hiroaki Kuroda

Shigetsugu Ohgi

\title{
Fibrillin gene (FBN1) mutations in Japanese patients with Marfan syndrome
}

Received: November 1, 1999 / Accepted: November 9, 1999

\begin{abstract}
Marfan syndrome (MFS; MIM \#154700) is a connective tissue disorder characterized by cardiovascular, skeletal, and ocular abnormalities. The fibrillin-1 gene (FBN1; MIM no. 134797) on chromosome 15 was revealed to be the cause of Marfan syndrome. To date over 137 types of FBN1 mutations have been reported. In this study, two novel mutations and a recurrent de-novo mutation were identified in patients with MFS by means of single-strand conformational polymorphism (SSCP) analysis. The two novel mutations are a 4-bp deletion at nucleotide 28202823 and a G-to-T transversion at nucleotide 1421 (C474F), located on exon 23 and exon 11, respectively. A previously reported mutation at the splicing donor site of intron 2 (IVS2 G + 1A), which is predicted to cause exon skipping, was identified in a sporadic patient with classical MFS.
\end{abstract}

Key words Marfan syndrome $\cdot F B N 1 \cdot$ Fibrillin- $1 \cdot$ Japanese - Mutation - Gene

H. Chikumi · K. Nagata

Third Department of Internal Medicine, Faculty of Medicine, Tottori University, Yonago, Japan

T. Yamamoto $(\bowtie) \cdot$ Y. Ohta $\cdot$ E. Nanba

Gene Research Center, Tottori University, 86 Nishi-machi Yonago, 683-8503 Japan

Tel. +81-859-8283; Fax +81-859-34-8284

e-mail: tyamamot@grape.med.tottori-u.ac.jp

H. Ninomiya

Department of Neurobiology, School of Life Sciences, Faculty of

Medicine, Tottori University, Yonago, Japan

K. Narasaki · T. Katoh · I. Hisatome

First Department of Internal Medicine, Faculty of Medicine, Tottori University, Yonago, Japan

K. Ono $\cdot$ N. Tanaka $\cdot$ H. Kuroda $\cdot$ S. Ohgi

Second Department of Surgery, Faculty of Medicine, Tottori

University, Yonago, Japan

\section{Introduction}

Marfan syndrome (MFS; MIM \#154700) is inherited in an autosomal dominant manner, with a prevalence of 4 to 6 per 100,000 (Pyeritz and McKusik 1979). In 1991, fibrillin-1 (FBN1; MIM no. 134797), on chromosome 15 was identified as a major disease-causing gene of MFS (Dietz et al. 1991; Tsipouras et al. 1992). FBN1 comprises 65 exons, and encodes a large glycoprotein, which is a ubiquitous component of 10- to 12-nm microfibrils of the extracellular matrix (Sakai et al. 1986; Pereira et al. 1993). Since the identification of $F B N 1$, over 137 types of $F B N 1$ mutations have been revealed that are widely distributed throughout the gene (Collod-Béroud et al. 1998). We analyzed the mutations of the FBN1 gene in Japanese patients with MFS, and we discuss the phenotype-genotype relationship.

\section{Patients and methods}

Patients

Patient 1 is a 45-year-old man who has skeletal manifestations of MFS, including scoliosis, pectus excavatum, and arachnodactyly. His height is within normal limits $(174 \mathrm{~cm} ;+0.64 \mathrm{SD})$. He has medium grade myopia without dislocated lenses. Both his father and an older brother have similar manifestations. At age 35 years, he developed aortic aneurysm and mitral prolapse and received a replacement of the aorta with a composite graft.

Patient 2, a 47-year-old woman, has familial MFS with mild manifestations of MFS. Her height is $162 \mathrm{~cm}(+0.82$ $\mathrm{SD})$. She presents with arachnodactyly and coloboma lentis. Her mother has similar skeletal manifestations. At age 44 years, she received an aortic root replacement for annuloaortic ectasia and aneurysm of the ascending aorta.

Patient 3 is a 46 -year-old man. His height is $178 \mathrm{~cm}$ $(+1.36 \mathrm{SD})$ and weight is $59 \mathrm{~kg}$. He has arachnodactyly and pectus excavatum. There is no obvious familial history of 
MFS. At age 43 years, he received aortic root replacement for annuloaortic ectasia and aneurysm of the ascending aorta.

Genomic DNA extraction and polymerase chain reaction (PCR) amplification

DNAs were extracted from peripheral blood by phenol/ chloroform methods. More than 100 normal control DNAs were obtained, using a MagExtractor (Toyobo, Osaka, Japan), from blood samples of Japanese volunteers and used for a population study. PCR amplification of the genomic DNAs was done using standard methods. The PCR primers were synthesized according to Nijbroek et al. (1995). Informed consent for genomic examinations was obtained from all patients.

Single-strand conformational polymorphism (SSCP) analysis

The PCR products were subjected to SSCP analysis (Orita et al. 1989; Yuasa et al. 1997). We used a minigel $(10 \mathrm{~cm} \times$ $10 \mathrm{~cm}$ ), and analyzed the samples under four different electrophoresis conditions, in a combination of two sets of gel mixtures $(12 \%$ polyacrylamide gel with or without $5 \%$ glycerol) and two temperature conditions $\left(4^{\circ} \mathrm{C}\right.$ or $\left.22^{\circ} \mathrm{C}\right)$. DNA bands were visualized by silver staining.

\section{DNA sequencing}

PCR products that gave aberrant bands on SSCP analysis were subcloned to pGEM-T vector (Promega, Madison, WI, USA). DNA sequences were determined with a Thermo Sequenase fluorescent labeled primer cycle se- quencing kit with Cy5-labeled forward or reverse primers (Amersham Pharmacia Biotech, Uppsala, Sweden). The samples were analyzed with an ALFred automatic sequencer (Amersham Pharmacia Biotech). At least ten independent clones of each PCR product were isolated and sequenced in both directions. The sequence data were analyzed with Genetyx-Mac computer software (Software Development, Tokyo, Japan).

\section{Results}

2820delAATG. SSCP analysis of exon 23 detected abnormal bands in patient 1 , and subsequent sequencing showed a 4-bp deletion (AATG) in about half of the clones (Fig. 1). The deleted region was nucleotides 2820-2823 in exon 23. This deletion is predicted to cause a frameshift from codon 941 and to make a premature stop codon at 19 amino acids downstream.

G1421T (C474F). Similarly, an abnormal band was detected on exon 11 in patient 2 . Sequence analysis revealed a G-to-T substitution at nucleotide 1421 in exon 11 (Fig. 2). This substitution is predicted to cause an alteration of cysteine to phenylalanine at codon 474 . This mutation was detected in none of the 100 unrelated Japanese volunteers.

IVS2 $G+1 A$. SSCP analysis of exon 2 showed an aberrant band in patient 3 , and subsequent sequencing confirmed a G-to-A transition at the intron 2 splicing donor site (IVS2 G + 1A) (Fig. 3). This mutation had been identified in a sporadic case reported by Dietz et al. (1993). They reported that this splicing donor site mutation resulted in a skipping of exon 2 (83-bp).
Fig. 1. A Single-strand conformational polymorphism (SSCP) analysis of exon 23 showing additional aberrant bands in patient 1 (C, Control). B Sequence analysis of a wild type allele (top) and a mutant allele (bottom) in patient 1 , showing a 4-bp deletion. Boxed nucleotides (AATG) are deleted

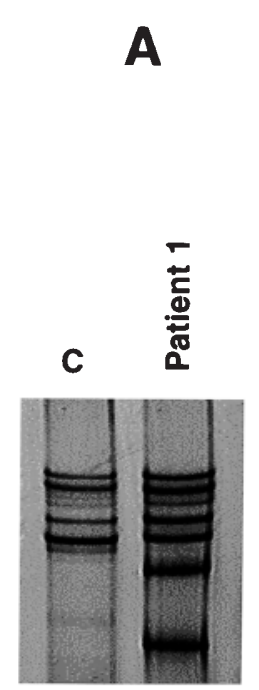

B

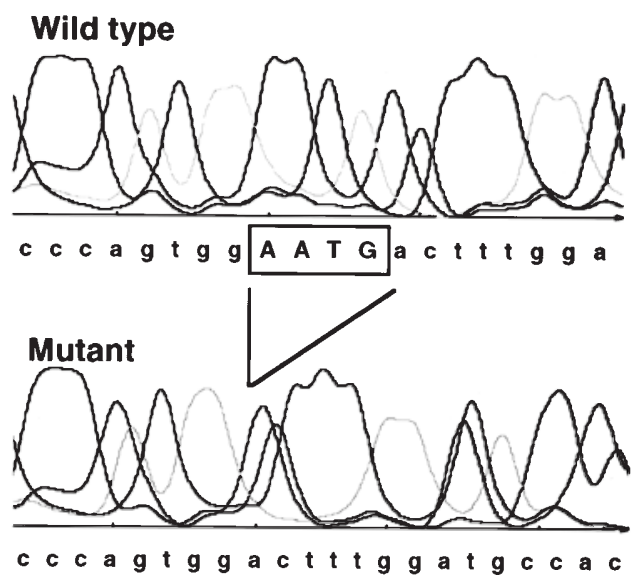


Fig. 2A,B. Results for patient 2 . A SSCP analysis of exon 11, indicating extra bands in patient 2 (C, Control). B Sequence analysis of a wild type allele (top) and a mutant allele (bottom) in patient 2 . The location of the G1421T missense transversion is indicated with the arrow

Fig. 3. A SSCP analysis of exon 2 shows aberrant bands in patient 3 (C, Control). B Sequence analysis of a wild type allele (top) and a mutant allele (bottom) in patient 3 . A G-to-A transition (shown by box) is located on a consensus splicing donor site
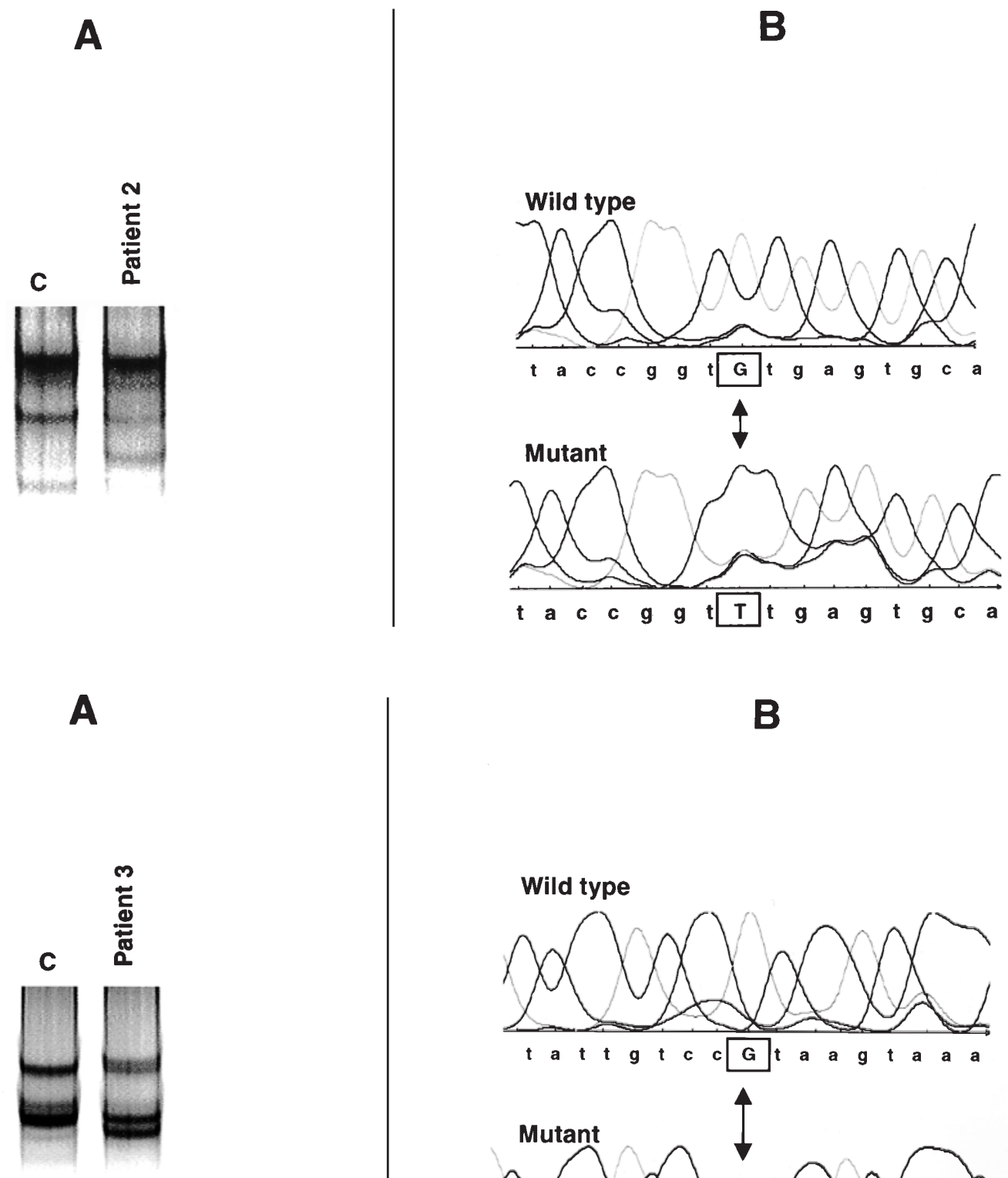

B
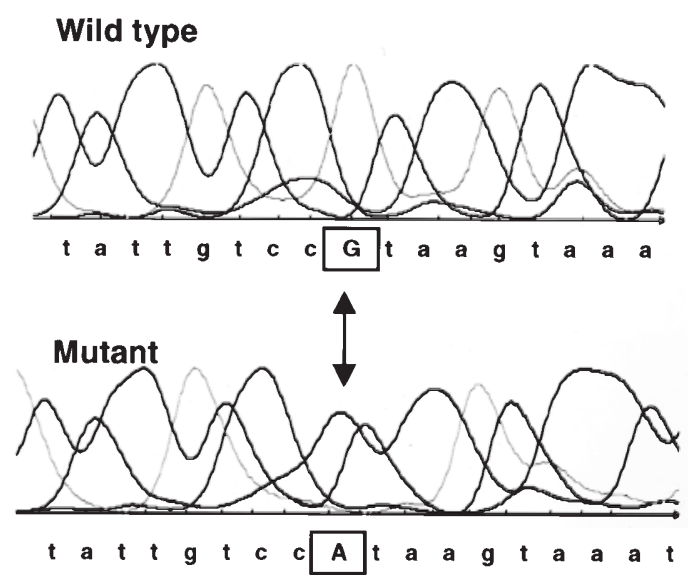

\section{Discussion}

A novel 4-bp deletion, nucleotides $2820-2823$ on exon 23 , was confirmed in patient 1 . The 4-bp deletion is predicted to cause a frameshift and generate a truncated fibrillin polypeptide. Generally, mutations that cause premature stop codons result in decreased levels of the mutant transcript, and, consequently, in a clinical phenotype milder than the that of classical MFS (Dietz et al. 1993; Aoyama et al. 1994). Because the manifestation in patient 1 is relatively mild, this patient's genotype and his phenotype are in accordance with this theory.

The G1421T transversion (C474F) detected in patient 2 causes the substitution of a conserved cysteine residue. Cys- teine in codon 474 forms an intradomain disulfide linkage with another cysteine (Dietz et al. 1992; 1993). This mutation is predicted to disrupt the disulfide linkage and to cause a severe phenotype. However, the phenotype in patient 2 is not so severe.

In patient 3 , a recurrent de-novo transition (IVS2 $\mathrm{G}+$ 1A) was identified. It is predicted that this splicing donor site mutation causes exon skipping, as in the case reported by Dietz et al. (1993). Although DNA samples from family members of patient 3 were unavailable, there was no family member with a manifestation of MFS. Therefore, patient 3 is thought to be a sporadic case of MFS caused by this recurrent de-novo mutation. Except for the ocular involvement, both patient 3 and Dietz's case are classified as classical type. 
In conclusion, two novel mutations (2820delAATG, G1421T) and a recurrent de-novo mutation (IVS2 G +1 A) in the FBN1 gene were identified in unrelated Japanese patients with MFS.

\section{References}

Aoyama T, Francke U, Dietz HC, Furthmayr H (1994) Quantitative differences in biosynthesis and extracellular deposition of fibrillin in cultured fibroblasts distinguish five groups of Marfan syndrome patients and suggest distinct pathogenetic mechanisms. J Clin Invest 94:130-137

Collod-Béroud G, Béroud C, Ades L, Black C, Boxer M, Brock DJ, Holman KJ, de Paepe A, Francke U, Grau U, Hayward C, Klein HG, Liu W, Nuytinck L, Peltonen L, Alvarez-Perez AB, Rantamaki T, Junien C, Boileau C (1998) Marfan Database (third edition): new mutations and new routines for the software. Nucleic Acids Res 26:229-233

Dietz HC, Cutting GR, Pyeritz RE, Maslen CL, Sakai LY, Corson GM, Puffenberger EG, Hamosh A, Nanthakumar EJ, Curristin SM, Stetten G, Mayers DA, Francomano CA (1991) Marfan syndrome caused by a recurrent de-novo missense mutation in the fibrillin gene. Nature 352:337-339

Dietz HC, Saraiva JM, Pyeritz RE, Cutting GR, Francomano CA (1992) Clustering of fibrillin (FBN1) missense mutations in Marfan syndrome patients at cysteine residues in EGF-like domains. Hum Mut 1:366-374
Dietz HC, McIntosh I, Sakai LY, Corson GM, Chalberg SC, Pyreritz RE, Francomano CA (1993) Four novel FBN1 mutations: singificance for mutant transcript level and EGF-like domain calcium binding in the pathogenesis of Marfan syndrome. Genomics $17: 468-475$

Nijbroek G, Sood S, McIntosh I, Francomano CA, Bull E, Pereira L, Ramirez F, Pyeritz RE, Dietz HC (1995) Fifteen novel FBN1 mutations causing Marfan syndrome detected by heteroduplex analysis of genomic amplicons. Am J Hum Genet 57:8-21

Orita M, Iwahana H, Kanazawa H, Hayashi K, Sekiya T (1989) Detection of polymorphisms of human DNA by gel electrophoresis as single-strand conformation polymorphisms. Proc Natl Acad Sci USA 86:2766-2770

Pereira L, D'Alessio M, Ramirez F, Lynch JR, Sykes B, Pangilinan T, Bonadio J (1993) Genomic organization of the sequence coding for fibrillin, the defective gene product in Marfan syndrome. Hum Mol Genet 2:961-968

Pyeritz RE, McKusick VA (1979) The Marfan syndrome: diagnosis and management. N Engl J Med 300:772-777

Sakai LY, Keene DR, Engvall E (1986) Fibrillin, a new 350-kD glycoprotein, is a component of extracellular microfibrils. J Cell Biol 103:2499-2509

Tsipouras P, Del Mastro R, Sarfarazi M, Lee B, Vitale E, Child AH, Godfrey M, Devereux RB, Hewett D, Steinmann B, Viljoen D, Sykes BC, Kilpatrick M, Ramirez F, and the international Marfan syndrome collaboratory study (1992) Genetic linkage of the Marfan syndrome, ectopia lentis, and congenital contractural arachnodactyly to the fibrillin genes on chromosomes 15 and 5. N Engl J Med 326:905-909

Yuasa I, Umetsu K, Vogt U, Nakamura H, Nanba E, Tamaki N, Irizawa Y (1997) Human orosomucoid polymorphism: molecular basis of the three common ORM1 alleles, ORM1*F1, ORM1*F2, and PRM1*S. Hum Genet 99:393-398 